\title{
THE DETERMINATION OF LOCAL TEXTURE BY ELECTRON DIFFRACTION-A TUTORIAL REVIEW
}

\author{
R. A. SCHWARZER \\ Institut für Metallkunde und Metallphysik der TU, Grosser Bruch 23, \\ D-3392 Clausthal-Z., Germany
}

\begin{abstract}
Electron diffraction methods are briefly reviewed for the determination of individual grain orientations and for the measurement of SAD pole figures. The standard techniques of orientation determination grain by grain using a TEM are the interpretation of selected area electron spot and microbeam Kikuchi diffraction patterns. Electron-transparent thin samples are required. Specimen regions smaller than $500 \mathrm{~nm}$ or $10 \mathrm{~nm}$ in diameter, respectively, can be studied. Alternatively, quantitative pole figures can be measured using a TEM from selected areas down to $0.5 \mu \mathrm{m}$ in diameter.

The orientations of grains in a bulk sample are obtained with an SEM from backscatter Kikuchi patterns or from (selected area) channelling patterns. Spatial resolution is approximately $1 \mu \mathrm{m}$ or $5 \mu \mathrm{m}$, respectively.

Individual grain orientation and pole figure measurements can be performed on-line by interfacing the electron microscope with a computer. In contrast to X-ray or neutron diffraction, electron microscopes give the means to image microstructural details at high resolution as well as to study crystal texture of exactly the same specimen region by electron diffraction.
\end{abstract}

KEY WORDS Electron diffraction, Kikuchi pattern, EBS pattern, channelling pattern, micro Kossel X-ray diffraction.

\section{INTRODUCTION}

The determination of crystal grain orientations and their statistical distribution in a polycrystal (crystal "texture", preferred orientations) is of great concern to material science. Several important material properties are anisotropic, i.e. they are dependent on crystal orientation, and texture may be changed specifically during production and use of a workpiece. Texture can conveniently be measured on a macroscopic scale using X-ray or neutron diffraction techniques. Often, however, microstructure is inhomogeneous on a microscopic scale, and material fails in consequence of local flaws. Hence the measurement of the texture of small regions or even the determination of individual grain or subgrain orientations is necessary. The knowledge of microscale texture may clarify the mechanisms of texture transformation during deformation and recrystallization, the formation of shear and deformation bands, and fracture processes. 
The outstanding advantage of transmission and scanning electron microscopy over light microscopy, X-ray and neutron diffraction is in texture analysis that a selected specimen region can both be imaged at high resolution and be investigated for its grain orientations. The fast but mainly qualitative methods of illustrating texture inhomogeneities by metallographic means, e.g. colour etch or grain-orientation specific etch preceding an investigation by light microscopy, can be supplemented by the following quantitative methods of local texture analysis:

- dark-field imaging in the TEM as a means of quantitative texture mapping.

- the measurement of pole figures from small regions by selected area diffraction with a TEM.

- the determination of single grain orientations by electron diffraction with a TEM or SEM, and the construction of the orientation distribution function or misorientation distribution function.

Various methods for the determination of crystal orientation have been developed since the early days of electron microscopy. Some of the first methods are based on trace analysis of orientation specific morphology in bright- or dark-field images, e.g. the arrangement of stacking faults, or habit, slip and twinning planes (von Heimendahl, 1964; Drazin and Otte, 1963; Crocker and Bevis, 1964). Trace analysis methods are limited to low-index planes and even so, the results may be ambiguous. Calculations become increasingly more complex with higher indices. The terms "bright-field" and "dark-field" refer to images to which the forward-scattered ("primary") beam does or does not contribute, respectively.

Crystal orientations may be determined most precisely with high-resolution transmission electron microscopy. The sites of atom columns in the crystal can be seen on an atomic scale in lattice plane interference micrographs (Figure 1) from which the crystal orientation can directly be deduced. This technique is particularly valuable for the study of orientation correlations and the microstructure of grain and phase boundaries. Crystal lattice imaging will, however, not become a routine method for local orientation measurement, due to the following limitations:

- Specimen thickness in the probed area must not exceed about $20 \mathrm{~nm}$. Sample preparation is difficult, and only small regions are suitable such that statistical sampling over a larger contiguous specimen seems hardly possible.

- Extremely thin specimens are not always a good representation of bulk material.

- Resolution of a modern TEM is limited to about $0.15 \mathrm{~nm}$ which is sufficient to only resolve the widest lattice spacings in metals, i.e. only low-index lattice planes are visible. Ionic and covalent crystals often have larger lattice parameters, and are thus better suited for lattice imaging.

- Extensive computer simulation calculations are necessary for a reliable image interpretation.

- Stringent conditions for high resolution microscopy must be fulfilled.

- A dedicated high-resolution TEM must be available. 

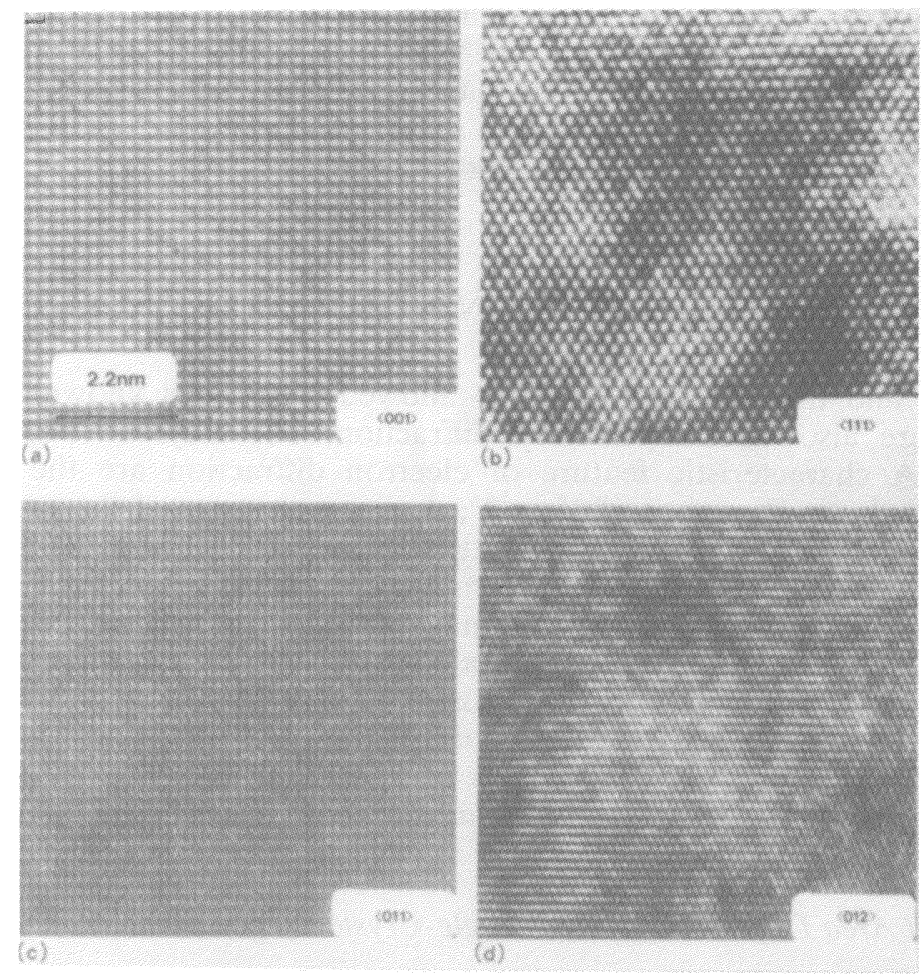

Figure 1 Lattice plane interference micrographs of the $b c c$ molybdenum structure (from: Bourret and Penisson, 1987). (a) $\langle 001\rangle$ projection showing two perpendicular sets of $\{110\}$ planes $(d=0.22 \mathrm{~nm})$. (b) $\langle 111\rangle$ projection showing three $60^{\circ}$ sets of $\{110\}$ planes. (c) $\langle 011\rangle$ projection showing one set of $\{110\}$ and one set of $\{200\}$ planes $(d=0.157 \mathrm{~nm})$. (d) $\langle 012\rangle$ projection showing one set of $\{200\}$ and two sets of $\{121\}$ planes $(d=0.128 \mathrm{~nm})$. Magnification is the same on the four micrographs.

The usual methods of individual grain orientation and local texture measurement are based on electron diffraction. Different types of electron diffraction patterns can be produced in the electron microscope that are:

- in transmission with a stationary primary beam ring patterns polycrystalline specimen texture patterns specimen with preferred orientations spot patterns $\}$ single-crystal area of specimen Kikuchi patterns

- in reflection from single-crystal region of a bulk sample channelling patterns -conventional scanning mode $\}$ scanning primary beam -rocking beam (SAD) mode backscatter Kikuchi patterns stationary primary beam (BKP; EBSP)

There is no strict attribution of the types of pattern to the main variants of electron microscopes, since with an appropriate attachment a TEM can be used in 
scanning (transmission) mode of operation (STEM) to produce channelling patterns and, on the other hand, an SEM may be operated as a diffractograph with stationary collimated beam to produce spot or ring patterns on a fluorescent screen beneath a transmissive specimen, or to produce Kossel or pseudo Kossel $\mathrm{X}$-ray diffraction patterns from small specimen regions.

\section{SELECTED AREA DIFFRACTION IN THE TEM}

Bragg's law and the selection rules for allowed reflections known from X-ray diffraction are also valid in electron diffraction, but they are not obeyed as rigorously. A characteristic feature of electron diffraction are the very small diffraction angles in the range of $1^{\circ}$ or $2^{\circ}$, as a consequence of the small electron wavelength as compared to the crystal lattice spacings. Hence diffraction spots are produced only by lattice planes whose common zone axis is virtually parallel to the primary beam, and a conventional electron diffraction pattern yields information about crystal texture only along a specimen direction close to the primary beam direction (Figure 2). Most methods of orientation analysis are based on strongly simplified models of diffraction and make merely use of the geometry of a pattern, i.e. the locations of maximum intensity. More elaborate techniques consider the locations and relative values of diffracted intensity.

\subsection{Selected Area Diffraction from a Single-Crystal Region}

A spot pattern is generated if a parallel electron beam falls on a single-crystal region of the specimen (Figure 2a). The diffraction pattern consists of bright regular spots which may be interpreted as being the intersections between the

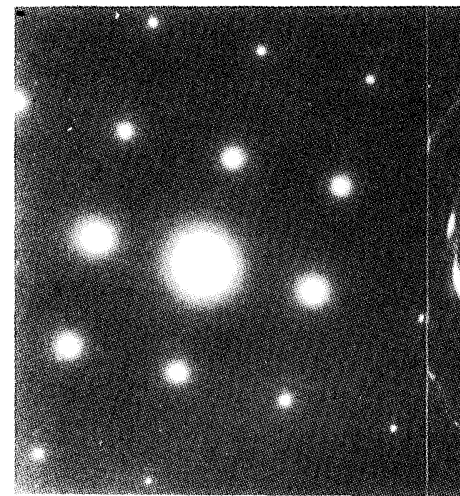

(a)

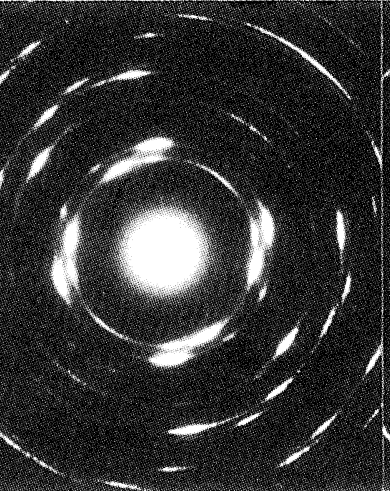

(b)

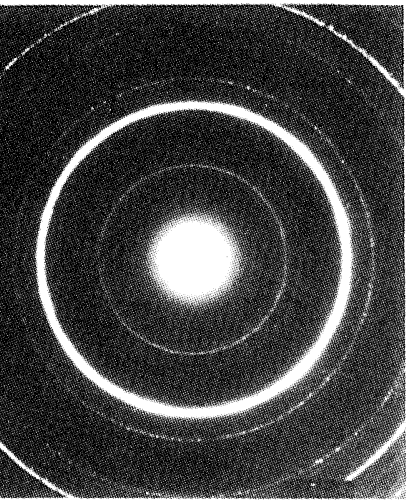

(c)

Figure 2 Selected area electron diffraction patterns of gold at $300 \mathrm{kV}$ accelerating voltage. (a) spot pattern form single-crystal area of specimen, $\langle 001\rangle$ projection. (b) texture pattern from specimen with $\langle 111\rangle$ fiber texture, fiber axis $55^{\circ}$ inclined to primary beam. (c) ring pattern from specimen with $\langle 111\rangle$ fiber texture, primary beam parallel to fiber axis. 


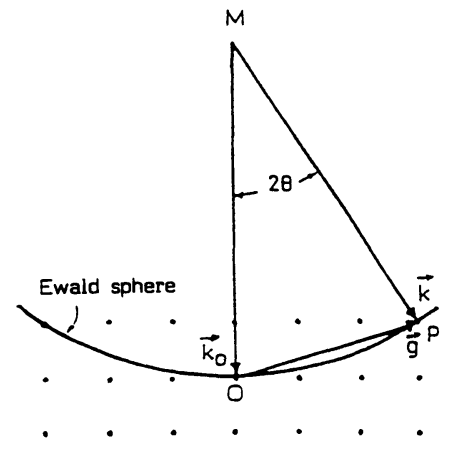

a) infinite crystal

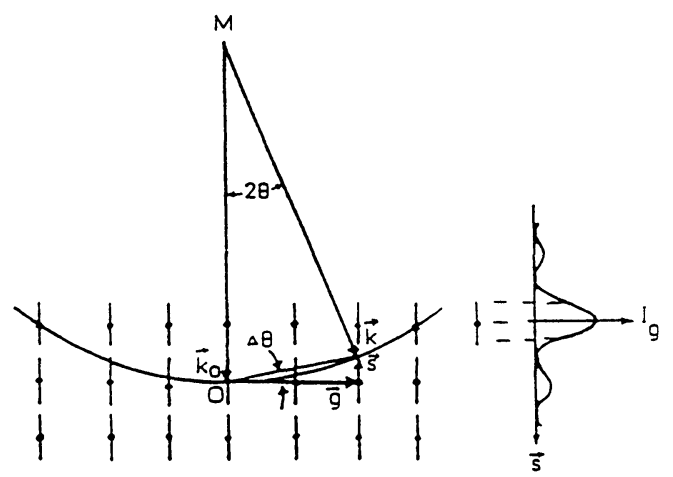

b) thin crystal

c) intensity along a spike

Figure 3 Ewald sphere construction in reciprocal space that illustrates the formation of a spot pattern.

Ewald sphere and the reciprocal lattice points of the crystallite. Since the sample must be rather thin to be transparent for electrons, the reciprocal lattice points are elongated along the foil normal to become "spikes" in reciprocal space (Figure 3). They may be intersected by the Ewald sphere and contribute intensity to the corresponding diffraction spots even though the Bragg condition is not exactly met. The consequence of the deviation is a predominance of low-index spots, and the primary beam direction is often mistaken for a low-index zone axis. Incorrect values of crystal orientations from spot patterns may give rise to false peaks on experimental pole figures (Haeßner et al., 1966). Real orientation distributions and particularly orientation relationships are thus obscured.

In selected area diffraction mode (SAD) of the TEM the specimen is illuminated with a parallel beam of electrons, and a region of interest is selected on the enlarged image, with the help of a diaphragm in the intermediate image plane. Finally the diffraction pattern from this area is displayed by focussing the back focal objective plane onto the final image screen. Spatial resolution in SAD cannot be improved to any extent by simply stopping down the size of the selected area. As a consequence of the spherical aberration of the objective lens and unavoidable focussing errors, the individual dark-field images, which are formed on the selector diaphragm by the diffracted beams, are slightly displaced with respect to each other and to the bright-field image. An additional displacement of the selected area in the specimen may result from transverse stray fields when switching the intermediate lens from imaging to diffraction mode (Riecke, 1961). In modern transmission electron microscopes the smallest selectable area in SAD is in the range of $0.2 \mu \mathrm{m}$ to $0.5 \mu \mathrm{m}$, as compared to about $1.5 \mu \mathrm{m}$ in older ones.

\subsection{How to Improve Spatial Resolution in the TEM}

Spatial resolution of orientation determination can be improved with standard transmission electron microscopes beyond the limits of selected area diffraction in 
three ways:

- In dark-field imaging mode of the TEM the specimen is tilted consecutively to angular settings such that each time the grain of interest attains maximum intensity which means the crystallite has come to a precise (two-beam) Bragg position. In the corresponding diffraction patterns the active diffraction spots are identified by their high brightness. From three not coplanar angular settings and knowing the indexing of the spots the orientation of the crystallite can be calculated with respect to a reference frame (Plege, 1983; Tambuyser, 1985; Möck, 1991).

- Slightly defocussed dark-field images simultaneously allow the determination of both the size and-similar as from a spot pattern-orientation of individual crystallites (Reinbold and Hoffmann, 1975). Each active diffraction spot $(h k l)$ produces a dark-field image of the grain of interest which is radially displaced from the location of the in-focus or bright-field image by a distance proportional to $\Delta f / d_{h k l}$. Here $\Delta f$ stands for the defocus setting. An annular aperture diaphragm is recommended for high contrast. The minimum grain size is about $2 \mathrm{~nm}$, and the azimuthal angle resolution of orientation in the range of $\pm 2^{\circ}$.

- Finally, convergent beam electron diffraction (CBED) can be used to improve spatial resolution. The fine focussed primary beam is directed on a grain of interest of a minimum size down to about $5 \mathrm{~nm}$. A TEM with three condenser lenses (microbeam diffraction; "Feinstrahlbeugung") (Riecke, 1962) or a scanning transmission attachment are required. Each reflection on the usual spot pattern is widened to a disc whose diameter can be controlled by the convergence angle of the primary beam. With increasing disc size the accuracy of orientation measurement is reduced. On the other hand, dark-field images of the irradiated specimen region are produced inside the diffraction discs when the pattern is imaged slightly out-of-focus above or below the back-focal plane of the objective lens. A great advantage of this method lies in the convenient performance of tilt experiments on small microstructural details like precipitates within the irradiated region or the study of orientation relationships between different grains or phases (Kesternich, 1982).

\subsection{Interpreting Spot Patterns}

An electron diffraction spot pattern corresponds, according to Ewald's construction, closely to a plane section through the reciprocal crystal lattice normal to the primary beam direction. Low-index reflections predominate such that in most cases the primary beam seems to be parallel to a low-index zone axis of the crystal. For simple crystal structures a spot pattern may readily be indexed by visual inspection or by comparison with a spot pattern map from literature (Edington, 1975).

Special procedures are required to interpret more complex patterns from crystals of low symmetry, or if more than one zone axis contributes spots to a pattern. In a first step the spacings $R_{i}$ between the diffraction spots and the center spot of the transmitted primary beam are matched with the lattice spacings, $d_{h k l}$, allowed by the structure factor of the actual type of crystal lattice. The camera constant, $\lambda \cdot L$, has to be known as a common scaling factor. $\lambda$ denotes the 
electron wavelength and $L$ the camera length. For high crystal symmetry it is convenient to cancel $\lambda \cdot L$ from calculation by comparing the ratios of spot spacings, $R_{i} / R_{j}$, since the number of possible $(h k l)$ spots is rather small and the ratios are a characteristic measure. Secondly the angles between the lines drawn from the center spot to the diffraction spots correspond to the angles between the normals to the lattice planes. By considering both conditions and taking into account reasonable limits of measurement errors for all spots, false $\{h k l\}$ values can be ruled out and consistent indexings are found.

Indexing is ambiguous, since spot patterns in general show a two-fold symmetry. Unless the common zone axis parallel to the primary beam has even symmetry, the specimen has to be tilted to another zone. Having also indexed the new diffraction spots, the correct indexing can be selected which must conform with the angles of tilt. A unique indexing, however, is readily possible if spots of more than one zone axis are present in a pattern (Ryder and Pitsch, 1968). The indexing of the common zone axis-which is close to the primary beam direction-follows from the vector product of two $(h \mathrm{kl})$ triples of indexed spots.

While the angular orientation around the primary beam is as precise as the spot positions can be measured on the pattern, the beam direction may be uncertain to about $\pm 5^{\circ}$. This can easily be demonstrated by inspection of a pattern on the microscope screen. If the sample is rotated around the primary beam, the diffraction spots rigidly follow the crystal lattice movement. If the specimen is tilted, however, there is initially no change of spot positions to be seen. With increasing angle of tilt some spots fade out, and finally other spots appear which belong to a new zone axis. In order to obtain a more precise beam orientation, the relative intensities of the spots have to be taken into account (method of intensity center) (Laird et al., 1966; von Heimendahl, 1980) or spots from more than one zone axis have to be considered (Ryder and Pitsch, 1968). These methods are increasingly time consuming and not well suited for routine work.

Indexing of spot patterns is a straight-forward though tedious procedure. With the availability of laboratory computers several programs have been written for plotting spot pattern maps and indexing spot patterns (Rhoades, 1975; Goehner and Rao, 1977; Fraundorf, 1981; Stadelman, 1987). A detailed description and the source code of the program XIDENT can be found elsewhere (Rhoades, 1970).

\subsection{Selected Area Diffraction from Polycrystals}

If the diffracting specimen region is a polycrystal, the spot patterns, one for each illuminated crystallite, superimpose on the screen with the center spot of the transmitted primary beam in common. For a sufficiently great number of crystallites and random orientation distribution around the primary beam, a ring pattern is formed (Figure 2c). Indexing is simple, since only the ring radii have to be assigned to the interplanar spacings, $d_{h k l}$, with $\lambda \cdot L$ as a scaling factor, in accordance with Bragg's equation and the structure factor of the crystal lattice. Ring patterns are most commonly used to discriminate phases of different crystal lattice symmetries or largely different lattice constants, and to calibrate the diffraction constant, $\lambda \cdot L$, of the microscope. 


\subsection{Selected Area Diffraction Pole Figures}

A texture pattern (Figure $2 b$ ) occurs if preferred grain orientations are found in the diffracting specimen region. The pattern consists of arced reflections which fill more or less the Debye Scherrer rings, according to the spread of grain orientations around the incident beam. The intensity disibution on a single diffraction ring corresponds to the pole-density distribution on a single reflection circle - which is here approximately a great circle-on the particular pole figure. The true texture of the work-piece can not be deduced from a single diffraction pattern, since only those preferred orientations are represented whose lattice planes are virtually parallel to the primary beam. Hence a series of diffraction patterns must be considered while tilting the sample through increasing amounts of angle.

The measurement of pole figures with a TEM is based on this feature of texture patterns (Humphreys, 1982; Schwarzer 1983). The sample is placed in the side-entry goniometer of the microscope such that a reference direction (e.g. the rolling direction) is parallel to the goniometer axis, and the TEM is switched to selected area diffraction mode (Figure 4). The sample is then tilted stepwise at small increments, $\Delta \beta$, around the goniometer axis which is perpendicular to the primary beam direction. The diffracted intensity may be measured by densitometry of the diffraction micrographs (Schwarzer, 1982), or by using a movable detector in the final image plane of the microscope. It is more elegant, however, to make use of the built-in beam deflection coils and to perform a direct on-line

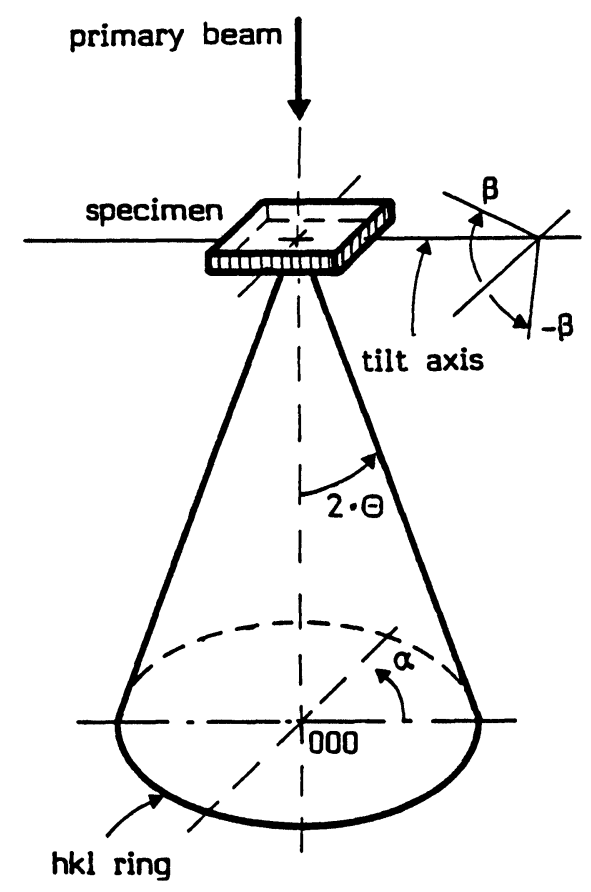

Figure 4 Schematics of set-up for SAD polefigure measurement. 
current measurement. Two types of deflection have been realized (Schwarzer, 1985):

- circular post-lens deflection of the texture pattern

- conical pre-lens deflection of the primary beam.

In the post-lens deflection mode, the diffraction pattern is circularly deflected such that a selected Debye Scherrer ring moves across a Faraday cup in the final image plane. Alternatively, the TEM can be switched to dark-field imaging mode with the aperture diaphragm centered on the back-focal point of the objective lens. When the incoming beam is deflected as to follow the surface of a cone, with its apex standing on the specimen and having a semi-vertex angle of twice the Bragg angle, the diffracted beams of the corresponding Debye Scherrer ring pass through the aperture diaphragm and leave the objective lens along the optic axis. Dark-field images are formed on the final image screen of a specimen region which is selected by the intermediate lens diaphragm. The bright shining grains fulfill the Bragg condition. The beam current on the final image screen is a measure of their volume fraction. In both modes of deflection the tilt angle, $\beta$, and the azimuth angle, $\alpha$, on the particular Debye-Scherrer ring define one point on the pole figure. For each setting of tilt angle the diffracted intensity values along a selected ring or in the dark-field images are measured on-line with the TEM under computer control.

Two correction procedures have been developed in order to interpret quantitatively the diffracted intensities in terms of pole-volume distribution (Schwarzer, 1983; Schwarzer 1991a). Allowance is made for the increase in diffracting volume and absorption when tilting the sample out of the direction of normal beam incidence. Since absorption increases unduly with steep inclination, the maximum angle of tilt ranges up to no more than $45^{\circ}$ or $60^{\circ}$, and incomplete pole figures are obtained. Post-lens deflection and intensity measurement from the diffraction pattern facilitates the separation of neighbouring diffraction rings, while conical pre-lens deflection allows the sampled specimen region to be observed in the dark-field images such that an unwanted image drift can be adjusted. Local texture can be measured down to the smallest specimen regions defined by selected area diffraction $(0.5 \mu \mathrm{m}$ diameter $)$.

The ODF of small regions can be calculated from measured SAD pole figures using inversion methods similar as with X-ray or neutron texture analysis. The blind areas in SAD pole figures differ from those in X-ray or neutron diffraction. An advantage is to apply an iteration method for pole-figure inversion whereby a constant but positive value is given to the immeasurable poles ("positivity method") (Dahms and Bunge, 1989). Materials of low crystal lattice symmetry, however, require increasingly more experimental pole figures to provide an accurate harmonic expansion of the ODF (Bunge, 1982), whereas with decreasing crystal symmetry the diffraction rings are closer spaced or even overlap in electron diffraction patterns, and less SAD pole figures can be measured separately. This means a severe restriction of selected area ODF analysis to cubic and hexagonal crystal symmetry. An application of selected area pole-figure measurement is discussed in a contribution to this volume (Weiland and Panchanadeeswaran, 1992). 


\section{MICROBEAM DIFFRACTION IN ELECTRON MICROSCOPES}

\subsection{Transmission Kikuchi Patterns}

Information about a very localized specimen region can be obtained with electron unlike X-ray or neutron diffraction, since the lens system of the electron microscope allow the electron beam to be focussed to a small spot selecting the sampled area. A Kikuchi pattern is produced, in analogy to Kossel X-ray patterns, if the illuminated crystal region has a low defect density and is sufficiently thick. The kinematic or the dynamic theories of electron diffraction are required for a detailed understanding of the formation of Kikuchi patterns, while the main features of the pattern geometry can be well interpreted by a simplified model (Figure 5) (Kikuchi, 1982): A portion of the electron beams (primary and diffracted) is scattered inelastically when they penetrate through the sample. The loss of energy by a single scattering event is very small and hence the increase in wavelength is negligible. These inelastically scattered electrons are present in all diffraction patterns producing a halo around the primary and diffracted beams. With increasing crystal thickness, however, they increase in intensity and finally fill a wide cone around the primary beam direction. Even if a selected lattice plane does not fulfill the Bragg condition for the primary beam, there are diffuse electrons which can be diffracted from either side of the lattice plane. They form ("Kossel") cones of excess and deficient intensity which intersect the recording plane in hyperbolas. Since electron wavelength is very small as compared to the lattice spacings, the Bragg angles, $\Theta$, are likewise small, and hence the semi-vertex angles of the Kossel cones are close to $90^{\circ}$. The traces of a pair of Kossel cones on the final image screen degenerate to form virtually a

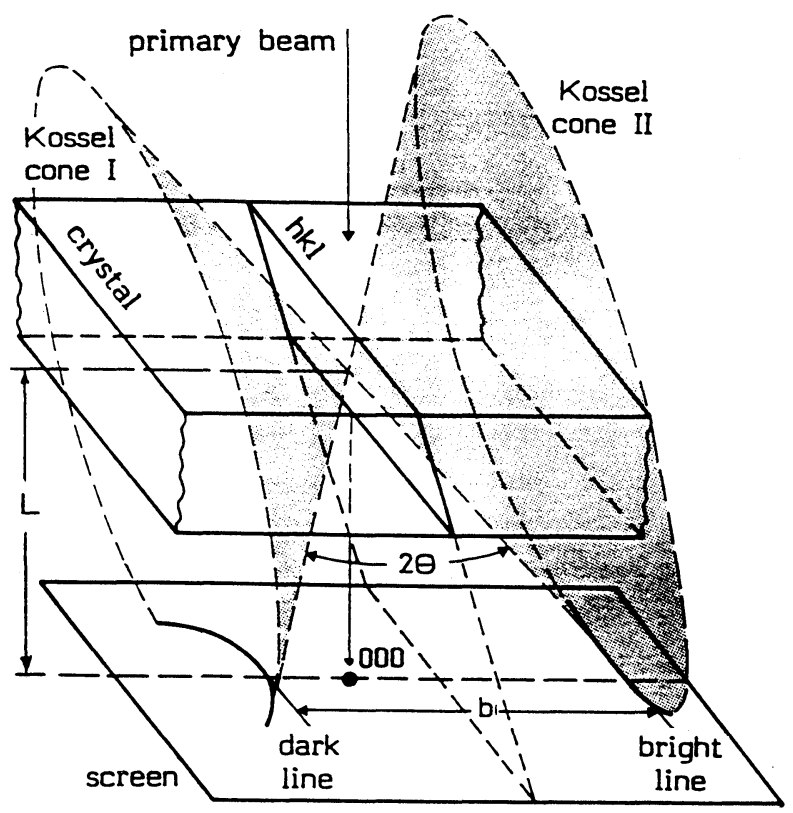

Figure 5 Kossel cones and Kikuchi lines (schematic). 
straight "dark" line near the primary beam and a parallel straight "bright" line further from the center. The region between two correlated Kikuchi lines is called a Kikuchi band. The intensity of bands may be higher or, under certain conditions, lower than the background of inelastically scattered electrons. The angular width of a band is twice the Bragg angle. The trace of the diffracting plane lies as a straight line almost midway between the Kikuchi line pair. Hence a Kikuchi pattern rigidly follows any movement, if the crystal is rotated around the primary beam or tilted. Accuracy of crystal orientation measurement is based on this feature and on the precision sharp Kikuchi lines can be located. If neighboring grains are studied, orientation differences less than $0.1^{\circ}$ can be detected. When determining the crystal orientation with respect to a reference frame fixed to the workpiece, a larger error than this may result from an inaccurate marking of the reference directions when taking the sample from the bulk material, from misalignments of the microscope and the specimen, and from local bending of the thin sample foil.

It is concluded from this model that clear Kikuchi lines are formed on two conditions: First, there must be a sufficient number of scattered electrons filling a large angular range around the primary beam direction. Second, there must be virtually the same crystal orientation over the whole diffracting sample volume, otherwise the particular Kikuchi patterns from local orientations would merge and fade out on the background. With samples made from real materials these conditions can be met by focussing the primary beam to a small probe on the sample area of interest such that the beam passes through a crystal volume of low local dislocation density. In practical work, the smallest probe size as well as spatial resolution are limited to about $10 \mathrm{~nm}$ due to the decrease in image brightness as the probe becomes smaller, and due to the spread of the scattered beam on its path through the sample foil. The demand for a perfect crystal is much alleviated, as dislocations often arrange themselves in networks. Annealing or creep deformation tend to produce a sub-cell structure where the dislocations cluster to form sharp boundaries around nearly dislocation-free cells of typically $1 \mu \mathrm{m}^{3}$ in size. When the sample is too thin to provide a scattering background sufficiently high for the formation of an intense Kikuchi pattern, the beam voltage may be decreased in order to enhance scattering. In general, however, the ability to study thick foils up to the limit of electron transparency is an outstanding benefit of the Kikuchi technique over spot patterns, since thick specimens are a better representation of bulk materials and sample preparation is less difficult. The convergence of the primary beam is of minor importance here, since the background of inelastally scattered electrons-which give rise to a Kikuchi pattern-spreads over an angular range about one order of magnitude wider than the primary beam aperture can be made.

\subsection{Backscatter Kikuchi Patterns}

In the SEM, Kikuchi patterns are generated by back-diffraction from a virtually perfect area of crystal (Venables and Harland, 1973). The stationary beam impinges on the bulk sample at a flat angle of typically $30^{\circ}$ from grazing incidence. For acquisition either a photographic plate or a fluorescent transmission screen is placed parallel to the incident beam, right in front of the tilted sample. In the latter arrangement the pattern may be recorded by a TV camera 


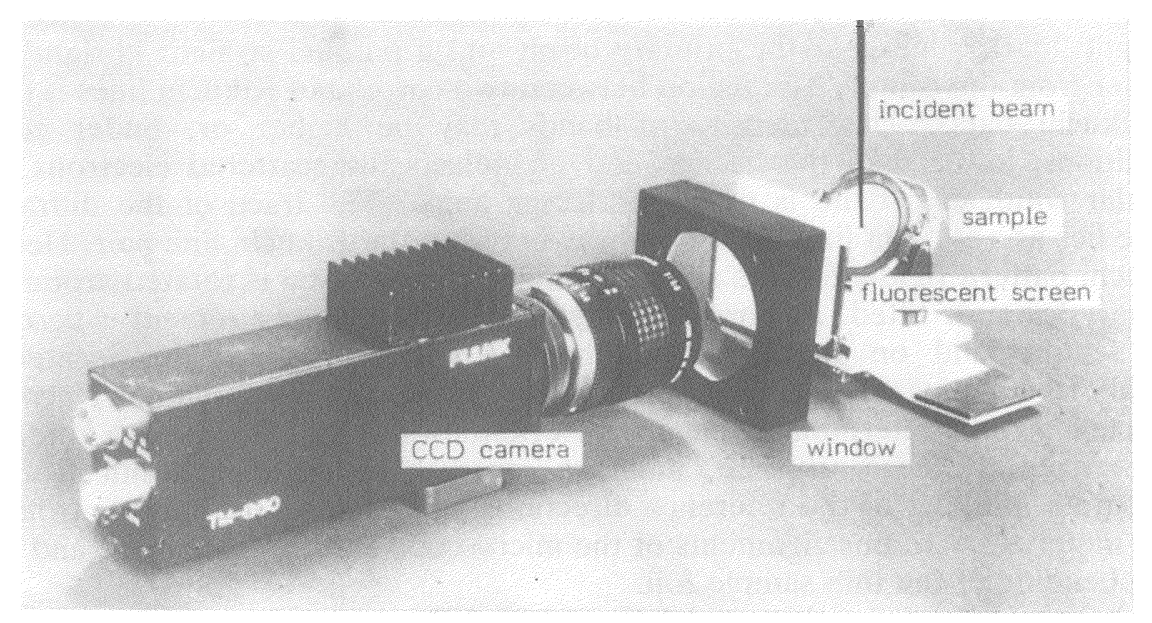

Figure 6 Set-up for the acquisition of backscatter Kikuchi patterns.

or by photography through a window from outside (Figure 6). An advantage over standard low light level TV cameras is to accumulate the pattern, without introducing distortions, on the sensor chip of a dedicated CCD camera, using Peltier cooling in order to reduce noise, and to transfer the digitized pattern in one frame scan to a computer for further interpretation (Schwarzer, 1989a).

Each pattern extends over a wide angular range in reciprocal space and includes several principal zone axes. Hence indexing may be performed simply by comparing the positions of bands and zone axes in the actual pattern with those of a Kikuchi map from literature. Sufficient detail can be obtained in the patterns to permit crystallographic point group and space group identification (Baba-Kishi and Dingley, 1989). Backscatter Kikuchi patterns when recorded on an SEM are also called electron backscattering patterns (EBS patterns) (Venables, 1973). They can by far not compete with transmission Kikuchi patterns, if high spatial resolution and accuracy of measurement are required. Spatial resolution is similar as with SEM micrography using backscattered electrons, since the primary electrons are back-diffracted from a similar interaction volume (Figure 7). Some disadvantages of the steep specimen tilt are the foreshortened beam spot on the sample as well as the foreshortened micrographs, and the need for another backscatter electron detector which has to be placed in the lobe of scattered electrons beneath the sample, in order to receive intensity sufficient for clear images of the tilted sample region. Patterns from grains $0.5 \mu \mathrm{m}$ wide have been reported (Dingley, 1984). Backscatter Kikuchi patterns, when recorded with a TV camera, are more diffuse and show less details than transmission Kikuchi or channelling patterns. Often individual Kikuchi lines are missing on the TV image which are supposed to border the bands at an angular width of twice the Bragg angle. Due to the low resolution of these wide-angle diffraction patterns, the accuracy of orientation measurement is limited to about $1^{\circ}$ to $2^{\circ}$. This is often sufficient for texture measurement. The study of misorientations or the distribution of special grain boundaries, however, demands for a higher accuracy of the individual crystal lattice orientations. 


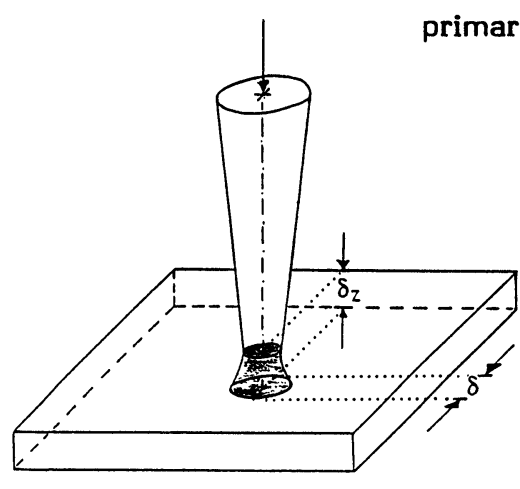

thin foil (TEM)

(a)

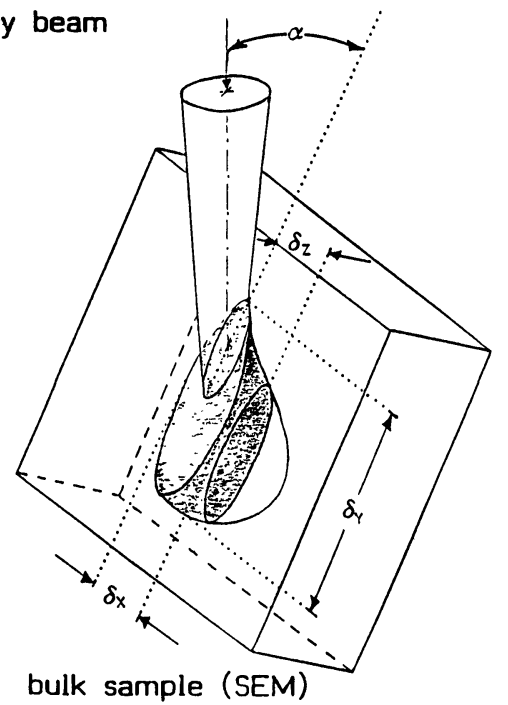

(b)

Figure 7 Diffraction volume and spatial resolution, $\delta$, with transmission (a) and backscatter (b) Kikuchi patterns.

\subsection{Channelling Patterns}

Channelling patterns from bulk samples are produced in the SEM by the variation of the backscattered intensity with the angle between the incident beam and the crystal lattice (Coates, 1967). As long as the primary beam strikes a low-index lattice plane at an angle less than the Bragg angle, an enhanced backscatter electron signal is produced, while intensity drops sharply when the Bragg angle is exceeded. For high-index lattice planes the dependence of intensity on the angle of incidence is more complex. As a rule, however, there is a significant change in intensity, whenever the incoming beam passes through the Bragg angle position (Figure 8). The signals can be collected, as in usual imaging mode, with a scintillator-multiplier or a solid state electron detector, amplified and displayed on the monitor screen of the SEM by modulating brightness. As the primary beam sweeps over some angular range, and the rocking angles are represented by linear $x$ and $y$ coordinates on the monitor screen, bright Kikuchi bands for low-index lattice planes and pairs of parallel Kikuchi lines at an angular width of twice the Bragg angle are formed. So the geometrical features of a channelling pattern are equivalent to those of a Kikuchi pattern.

Flat polished specimens are required for the standard raster scan channelling technique, and the diffracting region corresponds to the raster frame size on the sample surface. In the selected area mode of operation (van Essen and Schulson, 1969), the scanning facility is used in combination with the probe-forming lens to scan the angle of beam incidence, with the pivot point resting stationary on the sample surface ("rocking beam channelling pattern"). As a consequence of large spherical lens aberrations and the need for rocking angles in excess of $\pm 5^{\circ}$, the illuminated sample region can scarcely be made smaller than $10 \mu \mathrm{m}$ in commercial microscopes. This is not sufficient for the study of deformed materials since, 
Bloch waves type 1
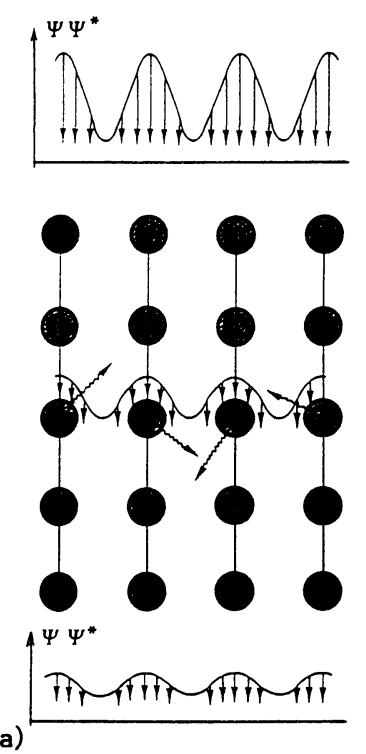

Bloch waves type 2
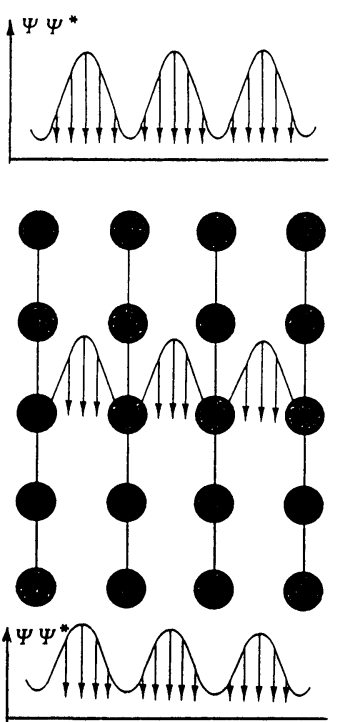
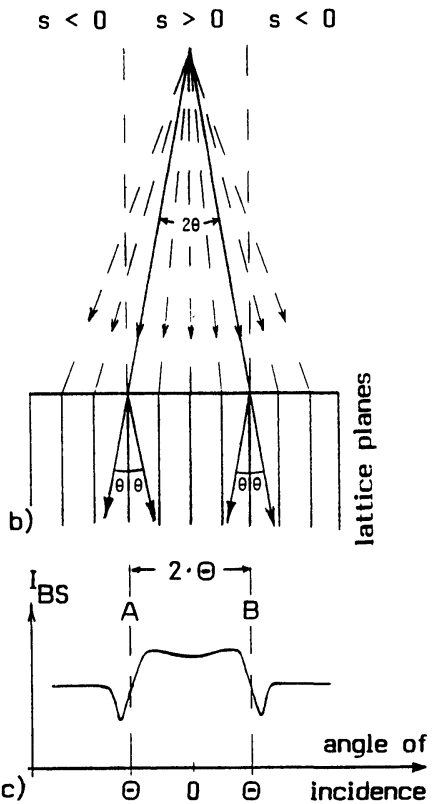

Figure 8 The formation of channelling patterns (schematic). (a) Bloch waves (type I and type II) propagating through the crystal lattice (b) variations of incidence angle with line scan (c) intensity profile across a channelling band.

with increasing dislocation density, the patterns soon become blurred, and interpretation is no longer possible. On the other hand, the sharpness of Kikuchi lines in the patterns provides a means of studying the local strain fields as well (Kaczorowski and Gerberich, 1991). Furthermore, the sharpness of Kikuchi lines is the convolution product of crystal perfection and primary beam aperture. So for a crisp channelling pattern it is necessary to keep the primary beam aperture smaller than about $10^{-3}$ rad. Some commercial SEM are equipped with the necessary lens and deflection coil controls and an additional diaphragm stage for diffraction.

Using a scanning transmission electron microscope (STEM) channelling patterns can be obtained in transmission mode by an angular scan of the beam over the specimen. Approximately the same spatial and angular resolutions are obtained as with microbeam transmission Kikuchi patterns.

\subsection{Micro Kossel X-Ray Diffraction}

The focussed primary beam of an SEM can be utilized for the micro Kossel diffraction technique (Dingley, 1978). The experimental situation is very similar to the formation of backscatter Kikuchi patterns. When the accelerating voltage is sufficiently high, characteristic X-ray radiation is emitted into all directions from the interaction volume of the primary beam with the sample. So virtually a point source of X-rays is formed. If X-rays strike a crystal lattice plane at the Bragg angle, they can be diffracted. This holds true for all beam directions around the 
lattice plane normal such that they form the surface of a "Kossel" cone with its apex centered on the interaction volume. Since the wavelengths of characteristic $X$-rays are in the range of the lattice spacings, the semi-vertex angles, $90^{\circ}-\Theta$, of Kossel cones may range from several degrees to a right angle. Usually a recording film is placed a few centimeters in front of the sample. The traces of the cones on the film are sharp conical sections rather than straight lines as with backsatter Kikuchi patterns.

In order to overcome the limitations with X-ray wavelengths characteristic for the sample material, the X-ray point source can be generated on the specimen surface in a thin layer of differently composed material (pseudo Kossel technique). If the anti-cathode film is placed some distance above the sample, a direct and possibly destructive interaction of the electron probe with the specimen can be avoided. Pseudo Kossel diffraction is then an extremely gentle method, since an X-ray beam rather than energetic electrons strikes the sample. The method can be applied for the study of sensitive or charging material. Spatial resolution, however, is impaired by projection. Micro Kossel diffraction is a tedious off-line technique, since long exposure times, the development of the film, and the measurement of cone positions are required. The interpretation of conical sections is more difficult than that of straight Kikuchi lines. On the other hand, the high accuracy of orientation measurement is only required for exceptional applications of texture analysis. Hence micro Kossel diffraction is mainly used for the precise determination of lattice parameters and internal stress, phase analysis, and the study of orientation differences. Spatial resolution is in the range of a few micrometers.

\section{ON-LINE MEASUREMENT AND INTERPRETATION OF ELECTRON DIFFRACTION PATTERNS}

Precise determination of crystal orientations from Kikuchi or channelling patterns is based on the sharpness of the lines and on the fact that they rigidly follow any rotation or tilt movement of the crystal (Figure 9). So the orientation of a grain can be obtained by simply identifying some Kikuchi lines or zone axes by comparison with a map or computer generated patterns. A significant advance is to perform measurement and interpretation of diffraction spot patterns (Carr, 1982), transmission Kikuchi or channelling patterns (Weiland and Schwarzer, 1985) and backscatter Kikuchi patterns (Dingley, 1989) on-line with a microscope interfaced with a computer.

With the SEM and STEM the positions of Kikuchi lines or zone axes on channelling or backscattering Kikuchi patterns can be determined from the monitor screen by moving the cursor on the selected points and reading its positions by the computer. In the same way a transmission Kikuchi pattern can be measured on the TEM by recording the pattern either from the screen through a window from outside (Figure 10) using a CCD TV camera, or by a dedicated TV camera incorporated in the microscope. An elegant method to measure positions on the TEM image screen, however, is to use the built-in deflection coils for shifting the diffraction pattern (or a micrograph when acquiring stereological data) across the screen such that successively selected points fall on a mark 


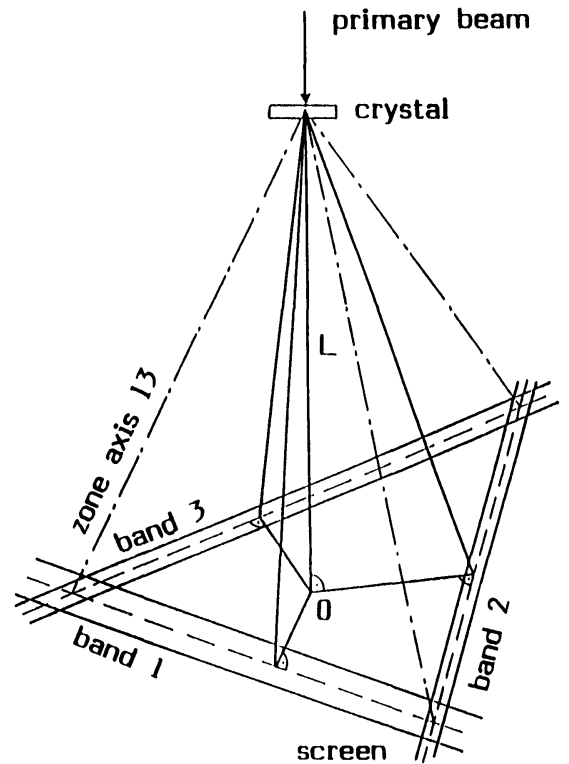

Figure 9 The geometry of Kikuchi patterns.

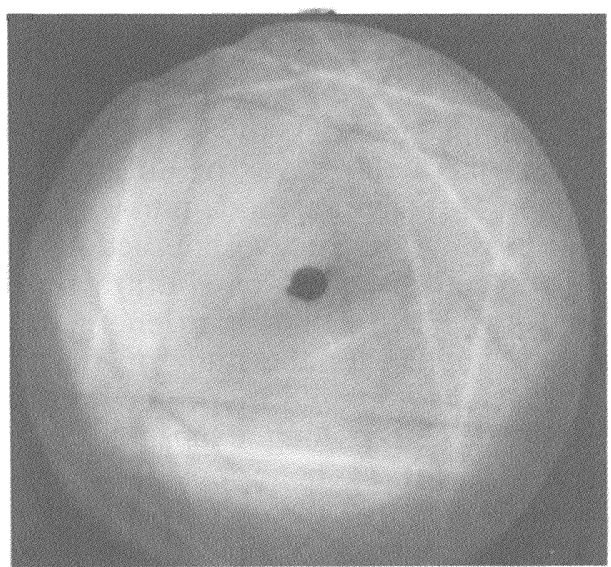

Figure 10 Transmission Kikuchi pattern of aluminum recorded from the final image screen of the TEM using a CCD camera. The center hole is made in the screen to enable SAD pole-figure measurement.

(Figure 11) (Weiland and Schwarzer, 1985). The necessary deflection voltages are transmitted to the computer as a measure of $x-y$ coordinates. There are only slight modifications in the TEM to be made. Details of a program for on-line interpration of Kikuchi patterns can be found elsewhere (Schwarzer, 1989b).

In order to exclude false orientations due to inaccurate measurement and to discriminate $[u v w]$ from $[\bar{u} \bar{v} \bar{w}]$ directions, it seems necessary to compute a Kikuchi map from each possible solution and to check it with the actual pattern on the microscope screen. Texture analysis by interpreting individual grain orientations is a question of statistics, and a large number of orientation values have to be collected. Great emphasis is laid on the development of fully automatic measurement techniques (Wright et al., 1991; Wright and Adams, 1992a). The advances with backscatter Kikuchi patterns is the topic of two contributions to this volume (Wright and Adams, 1992b; Juul-Jensen, 1992).

\section{REPRESENTATION OF INDIVIDUAL GRAIN ORIENTATIONS}

The orientation $g_{i}$ of a grain in a given reference frame is usually denoted (Bunge, 1982) by the Euler angles $\left(\varphi_{1}, \Phi, \varphi_{2}\right)$ (three independent angular parameters), or by the rotation matrix (array of nine parameters) which both specify a transformation of the crystallite frame into the reference frame. Alternative descriptions use Miller indices $(h k l)[u v w]$, particularly in the case of sheet metals, or Rodrigues vectors (Frank, 1988; Neumann, 1991). A complete 


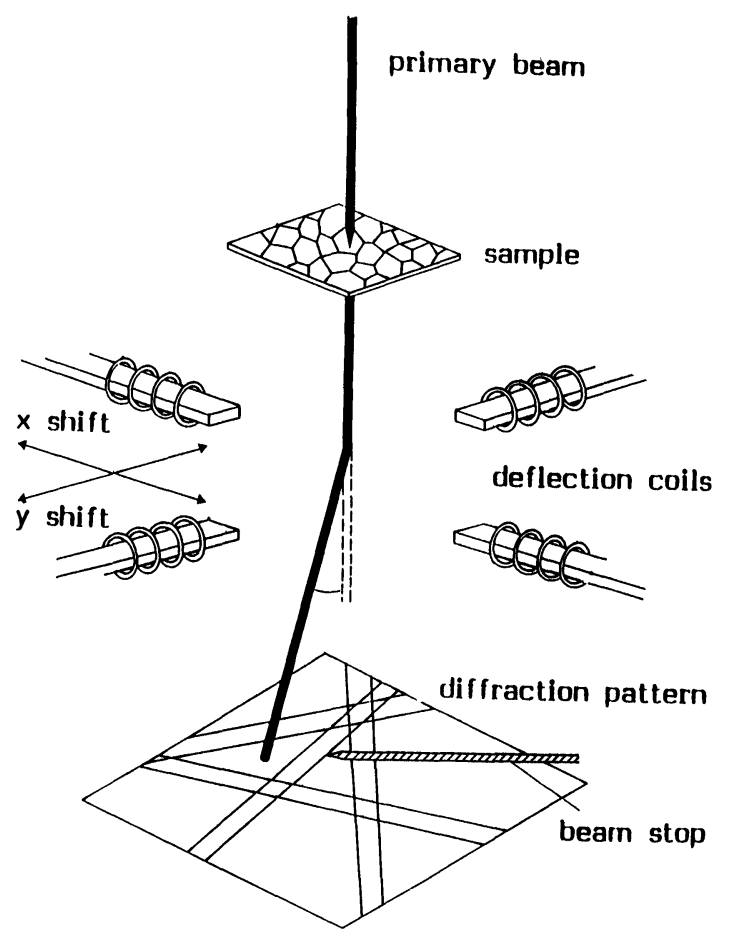

Figure 11 The on-line acquisition of Kikuchi patterns with a TEM.

representation of crystal texture is provided by the three-dimensional orientation distribution function (ODF) (Bunge, 1982). It describes the density of crystal lattice orientations as a function of the Euler angles. It is convenient to expand the ODF into a series of generalized spherical harmonics $\mathrm{T}(g)$. The expansion coefficients can be obtained either by the inversion of several experimental pole figures (Bunge, 1982), or by measuring the orientations and volumes of all individual grains in the sample area (Bunge, 1965; Wagner, 1986). Characteristic components of ordinary textures are already indicated by about 100 individual orientations, whereas weak textures may require a set of measurements of more than one order of magnitude larger (Wright and Adams, 1990).

In a similar way the distribution function of orientation differences, $\Delta g_{i}$, specified by Euler angles, can be obtained from individual orientation measurements by a series expansion of generalized spherical harmonics (Plege, 1987; Bunge and Weiland, 1988). The function is called misorientation distribution function (MODF) or orientation correlation function (OCF) between neighbouring grains (nOCF) or pairs of randomly located grains (rOCF) in a sample. When adjacent crystallites are considered, the density distribution of special grain boundaries is disclosed.

It is of advantage to condense the single orientation values in a graphical or statistical representation, since a polycrystal is in general composed of a great number of individual grains. For a rough estimate of crystal texture, two reference directions in the workpiece may be expressed by Miller indices with 
respect to the frames of the individual grains, and plotted point by point on a stereographic standard triangle to form inverse pole figures. Preferred orientations are thus indicated by clusters of points for a sufficiently great number of measured grains.

In addition to crystal texture, the correlation between spatial and orientation parameters in the sample is preserved by colour maps of the microstructure which look like stained glass windows. For instance, a look-up table can be constructed by an overlay of the colour triangle on the stereographic standard triangle, and each grain in the micrograph is given a colour specific for its orientation with respect to a reference direction in the workpiece. An example of this method (Inokuti et al., 1987) shows the spatial distribution of crystal orientations in grain oriented silicon steel after cold rolling for the sheet normal and for the rolling direction. The individual orientation measurement was performed using micro Kossel diffraction. 256 colours were available. The grain boundaries were classified to three categories according to the misorientations and depicted by three line widths in the maps. As an alternative, a complete spatial representation of grain orientations can be obtained in a single colour map by scaling each coordinate axis of a three-dimensional orientation space to the intensity of one of three basic colours (e.g. red, green and blue). With high-colour video graphic adaptors, more than 32,000 colours are available at $800 \times 600$ pixels on a standard personal computer, a number which seems large enough for a smooth colour shade. The Euler or particularly the Rodrigues orientation space can be used for the orientation-to-colour specification of individual grains in the map. Details of this method can be found elsewhere (Gerth et al., 1992). In a similar way the spatial distribution of anisotropic material parameters, deduced from individual grain orientation measurements as are the elastic energy per unit volume and square of strain, $u / \varepsilon^{2}$ or the shear stress per strain, $\sigma_{g} / \varepsilon$, can be represented by colour maps (Gerth et al., 1991). The merit of colour mapping is in that complex orientation correlations in space may become apparent at a glance.

\section{CONCLUSION}

Orientation measurements grain by grain yield information about the microstructure which is not obtained by any other technique. Direct access is given to the even and odd $C$ coefficients in the harmonic series expansion of the ODF. Furthermore correlations between spatial and orientation parameters can be investigated. Standard spot patterns are indispensable for the characterization of microstructure, e.g. for dark-field imaging, particulary with weak-beam technique, for the determination of dislocation density and Burgers vectors, and for the investigation of orientation relationships between second phases. Microbeam Kikuchi patterns play an outstanding role in texture analysis. Sample thickness is less critical. High spatial resolution facilitates the study of fine-grain materials, and orientation differences can be determined reliably due to the high accuracy of individual orientation measurement.

Channelling pattern technique is available on some commercial SEM as a built-in option. It is an adequate choice for the study of recrystallized, coarse grain materials like transformer sheet metal or semiconductor single crystals, and for the determination of local strain fields. Standard raster scan channelling mode 
is advantageous to the precise determination of orientation differences between neighbouring grains, since the patterns of both crystallites are displayed side by side in one micrograph at the same experimental settings, thus eliminating errors by misalignment. A backscatter electron detector is required for clear patterns as well as high orientation contrast between the grains in the micrographs.

Backscatter Kikuchi patterns in the SEM will cover most routine applications. Since measurement is performed on the surface of a bulk polycrystal, dynamic studies are possible, e.g. fracture or hot-stage experiments, grain growth, electromigration and chemical reaction processes. Furthermore large specimen

Table 1 Comparison of electron diffraction methods for local texture analysis and related measurements

\begin{tabular}{|c|c|c|c|c|}
\hline \multirow{2}{*}{$\begin{array}{l}\text { Type of } \\
\text { pattern }\end{array}$} & \multirow[t]{2}{*}{ Equipment } & \multicolumn{2}{|c|}{ Resolution } & \multirow[t]{2}{*}{ Main applications } \\
\hline & & Spatial & Angular & \\
\hline Spot pattern & TEM (SAD) & $0.5-1.5 \mu \mathrm{m}$ & $5^{\circ}\left(1^{\circ}\right)$ & $\begin{array}{l}\text { thin film samples } \\
\text { estimate of orientation } \\
\text { dark field imaging } \\
\text { dislocations (weak beam) } \\
\text { Burgers vector analysis }\end{array}$ \\
\hline \multirow[t]{2}{*}{$\begin{array}{l}\text { Transmission } \\
\text { Kikuchi } \\
\text { pattern }\end{array}$} & TEM (SAD) & $0.5-1.5 \mu \mathrm{m}$ & $0.1^{\circ}$ & $\begin{array}{l}\text { thick foil samples } \\
\text { coarse grains } \\
\text { crystal orientation } \\
\text { orientation difference } \\
\text { dislocation density }\end{array}$ \\
\hline & $\begin{array}{l}\text { TEM (micro- } \\
\text { beam) } \\
\text { STEM }\end{array}$ & $10 \mathrm{~nm}$ & $0.2^{\circ}$ & $\begin{array}{l}\text { fine grain structure } \\
\text { deformed materials } \\
\text { crystal orientation } \\
\text { orientation difference } \\
\text { grain growth }\end{array}$ \\
\hline $\begin{array}{c}\text { Backscatter } \\
\text { Kikuchi } \\
\text { pattern }\end{array}$ & $\begin{array}{l}\text { SEM \& } \\
\text { dedicated } \\
\text { attachment }\end{array}$ & $0.2-2 \mu \mathrm{m}$ & $1^{\circ}-2^{\circ}$ & $\begin{array}{l}\text { bulk samples } \\
\text { coarse grains } \\
\text { crystal orientation } \\
\text { fracture processes } \\
\text { dynamic studies (hot stage) } \\
\text { point groups, space groups }\end{array}$ \\
\hline $\begin{array}{c}\text { Channelling } \\
\text { pattern }\end{array}$ & SEM & $10 \mu \mathrm{m}$ & $0.5^{\circ}$ & $\begin{array}{l}\text { bulk samples } \\
\text { semiconductors } \\
\text { crystal orientation } \\
\text { orientation difference } \\
\text { internal stress, fracture } \\
\text { dynamic studies (hot stage) }\end{array}$ \\
\hline $\begin{array}{l}\text { Micro Kossel } \\
\text { pattern }\end{array}$ & $\begin{array}{l}\text { SEM \& } \\
\text { dedicated } \\
\text { attachment }\end{array}$ & $2 \mu \mathrm{m}$ & $0.4^{\circ}$ & $\begin{array}{l}\text { lattice parameters } \\
\text { internal stress } \\
\text { phase analysis } \\
\text { orientation differences } \\
\text { delicate samples }\end{array}$ \\
\hline $\begin{array}{l}\text { SAD pole } \\
\text { figure }\end{array}$ & $\begin{array}{l}\text { TEM \& } \\
\text { computer, } \\
\text { side-entry } \\
\text { goniometer }\end{array}$ & $0.5 \mu \mathrm{m}$ & & $\begin{array}{l}\text { thin-film samples } \\
\text { very fine-grain structure } \\
\text { high deformation } \\
\text { shear bands } \\
\text { texture fields }\end{array}$ \\
\hline
\end{tabular}


regions can be sampled at a rapid rate, and, by a successive removal of the surface, deeper volume layers can be measured in order to obtain a threedimensional texture analysis. An automated measuring procedure (Wright et al., 1991) will reduce the time for data collection drastically.

The lower accuracy and moderate spatial resolution obtained with backscatter Kikuchi or channelling patterns and the need for relatively low local deformation may lead to an arbitrary biasing of texture analysis by an unwanted selection of large or less deformed grains during measurement. The main drawbacks of TEM methods are the difficult sample preparation, particularly with multi-phase materials, and the limited field of view. Considering the high expense in sample preparation and equipment, the determination of individual crystal lattice orientations using electron diffraction will be reserved for special applications. Extremely fine-grain or strongly deformed materials are still not accessible to single orientation measurement, since the grains may not be distinguished from each other, or the patterns are too diffuse. Furthermore sample thickness in the TEM or the depth of information on bulk samples in the SEM must not exceed the actual grain size, otherwise the patterns from successive grains will merge and interpretation is hindered.

SAD pole figure measurement is the fastest method, if a spatial correlation between individual grains is not intended and pole figures or orientation distribution functions are sufficient for texture interpretation. It is the only local texture measure for thin films and extremely fine-grain or deformed materials when individual orientation measurement fails to produce clear patterns.

Electron diffraction techniques for texture analysis are complementary to each other and to standard X-ray pole figure measurement. There is no universal method, and the choice depends on the sampled grain structure as well as on the degree of information necessary for texture analysis. A comparison of the methods is given in Table 1 (Schwarzer, 1991b). Diffraction techniques for the SEM are compared in (Dingley, 1981).

\section{Acknowledgment}

The reprint permission of Figure 1 is gratefully acknowledged to A. Bourret and J.-M. Penisson, DRFMC/SP2M/S, CEN Grenoble, France.

\section{References}

Baba-Kishi, K. Z. and Dingley, D. J. (1989). Scanning 11, 305-312.

Bourret, A. and Penisson, J.-M. (1987). JEOL News 25E, 2-7.

Bunge, H. J. (1965). Z. Metallkunde, 56, 872-874.

Bunge, H. J. (1982). Texture Analysis in Materials Science-Mathematical Methods. Butterworths, London.

Bunge, H. J. and Weiland, H. (1988). Textures and Microstructures 7, 231-263.

Carr, M. J. (1982). JEOL News 20E, 35-38.

Coates, D. G. (1967). Phil. Magazine 16, 1179-1784.

Crocker, A. G. and Bevis, M. (1964). Phys. Stat. Sol. 6, 151-162.

Dahms, M. and Bunge, H. J. (1989). J. Appl. Cryst. 22, 439-447.

Dingley, D. J. (1978). Scanning Electron Microscopy 1978/I, 869-886.

Dingley, D. J. (1981). Scanning Electron Microscopy 1981/IV, 273-286, 258.

Dingley, D. J. (1984). Scanning Electron Microscopy 1984/II, 569-575.

Dingley, D. J. (1989). Proc. EMAG-MICRO 89; Inst. Phys. Conf. Ser. No. 98, pp.473-476. 
Drazin, M. D. and Otte, H. M. (1963). Phys. Stat. Sol. 3, 824-858.

Edington, J. W. (1975). Practical Electron Microscopy in Materials Science. Monograph Two: Electron Diffraction in the Electron Microscope. N.V. Philips' Gloeilampenfabrieken, Eindhoven.

van Essen, C. G. and Schulson, E. M. (1969). J. Material Science 4, 336-339.

Frank, F. C. (1988). Proc. 8th Intern. Conf. on Textures of Materials (ICOTOM 8). Kallend, J. S. and Gottstein, G. (Eds.). The Metallurgical Society, 1988, p. 3-13.

Fraundorf, P. (1981). Ultramicroscopy 6, 227-235.

Gerth, D., Katzer, R. and Schwarzer, R. (1991). Proc. Intern. Conf. on Grain Growth in Polycryst. Materials, Rome (in press).

Gerth, D., Zaefferer, St. and Schwarzer, R. A. (1992) (to be published).

Goehner, R. P. and Rao, P. (1977). Metallography 10, 415-424.

Haeßner, F., Jakubowski, O. and Wilkens, M. (1966). Mater. Sci. Eng. 1, 30-41.

von Heimendahl, M. (1964). J. Appl. Phys. 35, 457-458.

von Heimendahl, M. (1980). Electron Microscopy of Materials, An Introduction. Academic Press, New York.

Humphreys, F. J. (1983). Textures and Microstructures 6, 45-62.

Inokuti, Y., Maeda, Ch. and Ito, Yo (1987). Transactions of the Iron and Steel Institute of Japan, 302-311.

Juul-Jensen, D. (1992). Textures and Microstructures 20, 55-65.

Kaczorowski, M. and Gerberich, W. W. (1991). J. Materials Science 26, 1910-1918.

Kesternich, W. (1982). Proc. 10th Intern. Congress on Electron Microscopy, Hamburg 1982, Vol. 1, pp. 657-658.

Kikuchi, S. (1928). Jap. J. Phys. 5, 83-96.

Laird, C., Eichen, E. and Bitler, W. R. (1966). J. Appl. Phys. 37, 2225-2231.

Möck, P. (1991), Cryst. Res. Techn. 26, 653-658.

Neumann, P. (1991). Steel Research 62, 560-566.

Plege, B. (1983). Zur Entstehung der Primärrekristallisationstextur von CuZn30-Blechen. PhD Thesis, TU Clausthal.

Plege, B. (1987). In: Theoretical Methods of Texture Analysis, Ed.: H. J. Bunge; DGM Informationsges. Oberursel, 393-403.

Reinbold, M. and Hoffman, H. (1975). Methodensammlung der Elektronenmikroskopie 4.3.2, Wiss. Verlagsges. Stuttgart.

Rhoades, B. L. (1970). Research Report 70/76. Dept. of Mechanical Engineering, University of Canterbury, Christchurch, N.Z.

Rhoades, B. L. (1975). Micron 6, 123-127.

Riecke, W. D. (1961). Optik 18, 278-293.

Riecke, W. D. (1962). Optik 19, 19-116.

Ryder, P.-L. and Pitsch, W. (1968). Phil. Mag. 18, 807-816.

Schwarzer, R. A. (1982). Proc. 10th Intern. Congr. on Electron Microscopy, Hamburg 1982, pp. 129-130.

Schwarzer, R. A. (1982). Z. Metallkunde 73, 495-498.

Schwarzer, R. (1983). Beitr. elektronenmikroskop. Direktabb. Oberfl. (BEDO)16, 131-134.

Schwarzer, R. (1985). BEDO 18, 61-68.

Schwarzer, R. (1989a). BEDO 22, 279-282.

Schwarzer, R. (1989b). Die Bestimmung der lokalen Textur mit dem Elektronenmikroskop; Habil, Thesis, TU Clausthal.

Schwarzer, R. A. (1991a). In: Bunge, H. J. and Esling, C. (Eds.): Advances of Quantitative Texture Analysis. DGM Informations-Ges. Oberursel, pp. 51-72.

Schwarzer, R. A. (1991b). Steel Research 62, 542-547.

Stadelman, P. A. (1987). Ultramicroscopy 21, 131-146.

Tambuyser, P. (1985). Metallography 18, 41-49.

Venables, J. A. and Harland, C. J. (1973). Phil. Mag. 27, 1193-1200.

Wagner, F. (1986). In: Bunge, H. J. (Ed.): Experimental Techniques of Texture Analysis. DGM Informations-Ges. Oberursel, p. 115-123.

Weiland, H. and Panchanadeeswaran, S. (1992). Textures and Microstructures 20, 67-86.

Weiland, H. and Schwarzer, R. (1985). BEDO 18, 55-60.

Wright, St. I. and Adams, B. L. (1990). Textures and Microstructures 12, 65-76.

Wright, St. I., Zhao, J.-W. and Adams, B. L. (1991), Textures and Microstructures 13, 123-131.

Wright, St. I. and Adams, B. L. (1992a), Metallurgical Transactions 23A, 759-767. 